MATEC Web of Conferences 22,01023 (2015)

DOI: $10.1051 /$ matec conf/ 20152201023

(C) Owned by the authors, published by EDP Sciences, 2015

\title{
Assessing the Effect of Game System for Rehabilitation on Rehabilita- tion of Autism and Cerebral Palsy
}

\author{
Qunxia $\mathrm{Xu}^{*}$, Longxiang Chen, Tingfei Zhu \& Yun Xu \\ Department of Psychology, Zhejiang University of Technology, Hangzhou, Zhejiang, China
}

\begin{abstract}
This work presented a system to encourage children and adolescents with autism and cerebral palsy (CP) to improve their abilities of self-care, mobility and sociality. A study was conducted with 51 children with autism and 36 children with CP using the Game System for Rehabilitation with Kinect at intervals of one month. The scope of this study was aimed to evaluate the effects of Game System for Rehabilitation in terms of self-care, mobility and social function of children and adolescents with autism and CP. Pediatric Evaluation of Disability Inventory (PEDI) was used to determine the functional abilities of self-care, mobility and sociability. Data obtained allowed us to conclude that the positive effects of the Game System for Rehabilitation on the functional abilities of self-care, mobility and social function with disabilities were significant. The Game System for Rehabilitation applied for children and adolescents with autism and CP was effective for the targeted population. And it gives a new light for children with autism and CP to continue their rehabilitation successfully.
\end{abstract}

Keywords: game system for rehabilitation; Cerebral palsy (CP); autism; PEDI; rehabilitation; Kinect

\section{GENERAL INSTRUCTIONS}

\subsection{Background}

Autism and cerebral palsy are two kinds of the major causes of disability in the world.

Autism is basically characterized by social communication disorder, language development disorder, narrow area of interest, and stereotyped and repeated behavior to different extents, and it is increasing in the worldwide. There is a social impairment: the person with autism finds it hard to associate and empathize with other people. And there is communication impairment: the person with autism finds it hard to understand and use verbal and nonverbal communication. Also, there is a tendency to rigidity and inflexibility in thinking, language and behavior (Moore et al. 2010).

The cerebral palsy (CP) is defined as a developmental disability with restriction on motion, posture, and activities due to non-developmental physical changes followed by diseases that occurring in the brain of children those who are physically growing (Kim et al. 2012). In our country, the incidence of cerebral palsy is $1.8 \%-4 \%$. The motor disorders of individuals with cerebral palsy are often accompanied by loss of functionality and dependence on others in daily activities such as hearing disorder and language disorder (de Mello et al. 2005). These kinds of impairments are similar with autism and the causes of both diseases have something to do with the brain.

Rehabilitation can help patients with autism and cerebral palsy relieve sufferings and improve social skills. Previous studies have reported several ap-

*Corresponding author: xuqunxia@aliyun.com proaches to solve disabilities. The use of the combination of music and dance movement as a therapy on rehabilitation showed positive results on social relationship and improved communication skills. Several types of robots have been developed with great variations in design, size, and style to meet the rehabilitation of autism and CP (Miskam et al. 2014).

\subsection{New technologies on rehabilitation}

Conventional rehabilitations with physiotherapists are labor-intensive, expensive and lack of objective assessment as well as quantitative diagnosis and evaluations (Yeong et al. 2009).

Compared with the traditional methods of rehabilitation training, the use of virtual reality (VR) and video-games in rehabilitation training seems to be more effective on patients with autism or $\mathrm{CP}$ (Park et al 2011). The new technologies, such as virtual reality (VR), may offer the possibility of providing pro-grams of simpler exercises for children to pursue in-dependently (Burke et al. 2009).

First of all, game play makes therapy more exciting (Winkels et al. 2012), which can be regarded as an efficient method (Shane \& Albert 2008). Every child loves to play games. It partially follows the pattern of repetitive and stereotyped behavior of children with autism, which will definitely make them react and not immediately reject this new method of education (Boutsika, 2014). Through the game we can get in contact with the children with ASD and help them to shape social behavior (Kroustallakis, 2005). The patients upon completion of the game will gradually become familiar with the learning data and ideas, with the tools that will help them to learn and will increase 
the feeling of self-esteem, self-understanding, autonomy and independence. In addition, through the game, children with autism will become familiar with various objects and concepts that will be slowly incorporated in their daily lives (Kroustallakis, 2005).

What's more, the use of computer generated virtual environment does not include the face-to-face communication, which reduces the social interaction (Silver \& Oakes 2010). It's a method to alleviate some special populations, such as autism patients' anxiety (Kandalaft et al. 2013), and help them to train better.

Furthermore, computer games applied to autistic children must be sufficiently flexible to be adapted to the requirements of each child and integrate the personal data of its own world attached to its beliefs (Sehaba et al. 2005). Rehabilitation based on related technology research and development of computer game provides the special groups a standardized, predictable and controllable environment. They can be trained based on their ability (Golan \& Baron, 2006).

At last, video-games for activities have been de-scribed as 'one of the most innovative and promising recent developments in rehabilitation technology' (Weiss, 2004). Games make it possible to conduct the rehabilitation training at home instead of non-motivational typical movement therapy in clinic (Burdea et al. 2013). That saves a lot of time and expenses of children's families.

\subsection{Kinect as a useful tool to support rehabilitation}

Novel tracking devices, such as Nintendo Wii and balance board and Microsoft Kinect, have created the possibility to do rehabilitation at home where patients are guided by video-games (Wainer \& Ingersoll, 2011).

Nowadays, low-cost equipment which can be applied to rehabilitation is not so frequent, but Microsoft's Kinect is really one of a kind. Its advent was known as The Third Revolution In the $\mathrm{Hu}$ man-computer Interaction. The use of innovative computer technology is a promising strategy for delivering direct intervention to children and adults with autism or CP (Wainer \& Ingersoll, 2011). A drawback with motion sensors is that people have to fasten them on limbs, hold them in the hands or even wear them on the body to detect motions and possibly generated force feedback. Wearing sensors can also cause inconvenience and discomfort (Chang et al. 2013). Microsoft Kinect is a webcam-style add-on peripheral intended for the Xbox 360 game console. Kinect enables users to control and interact with the game console through a natural user interface using gestures without the need to touch a game controller, (Chang et al. 2013). Using Kinect means that the users need not be bothered with body sensors and that the system can save the users from wearing sensors that can be intrusive (Chang et al. 2013). The system includes an interactive interface with audio and video feedbacks to enhance motivation, interest, and perseverance to engage in physical rehabilitation. Details of rehabilita- tion conditions are also automatically recorded in the system, allowing therapists to quickly review rehabilitation progress. Therefore, this system reduces staff workload, helps to assess the quality of physical exercise, and enhances the efficiency of rehabilitation which increases the ability to perform daily tasks independently (Chang et al. 2013).

\section{METHODS}

\subsection{Subject}

In this study, 51 children with autism and 36 children with $\mathrm{CP}$ were randomly selected by special-education teachers, rehabilitation trainers and experts in the field of disabled rehabilitation as the study subjects. These 87 subjects were 3-19 years old and able to walk independently. And Table 1 presents the demographic information of subjects including gender, age and so on. More than 20 communities, special-education schools and hospitals in Hangzhou, Shaoxing, Taizhou, Ningbo, Huzhou and other cities of Zhejiang province are involved in this study.

Table 1. General information of objects.

\begin{tabular}{lllllll}
\hline $\begin{array}{l}\text { Disorder } \\
\text { type }\end{array}$ & $\mathrm{N}$ & & \multicolumn{2}{l}{ Gender } & Age & \\
\cline { 3 - 7 } & & Male & Female & M \pm SD & Min & Max \\
\hline $\begin{array}{l}\text { Autism } \\
\text { Cerebral }\end{array}$ & 51 & 40 & 11 & $8.92 \pm 3.38$ & 3 & 18 \\
palsy & 36 & 25 & 11 & $9.57 \pm 4.17$ & 3 & 19 \\
Total & 87 & 65 & 22 & $9.19 \pm 3.73$ & 3 & 19 \\
\hline
\end{tabular}

\subsection{Measurements}

The whole assessment of Game System for Rehabilitation consists of two parts.

First, Pediatric Evaluation of Disability Inventory (PEDI) was used as a structured report on parents or teachers in hospital, family or educational setting to measure children or adolescents' functional behavior, which is the best measure of activities of daily living (ADL) capability. The point is to test intervening effect of the Game System for Rehabilitation through a pretest-posttest design. The content of PEDI samples is in the domains of self-care, mobility and social function. These content domains are evaluated by three separate scales: (1) functional skill/behavior scale, (2) caregiver assistance scale, and (3) environment modifications and equipment. Hereby, the PEDI is made up of three parts: (1) 177 functional skill items $\quad$ (self-care $=58, \quad$ mobility $=54$, social function $=65$ ) rated 0 (incapable) or 1 (capable); (2) 16 items rated for caregiver assistance (5-point ordinal scale); (3) 16 items rated for environment modification (none, child-oriented, rehabilitation equipment, extensive). PEDI has good reliability and validity: internal consistency $\alpha=0.98 \sim 0.99$, test- 
retest $=0.98$, which is suitable for evaluation in the study (James, S. et al. 2014).

Second, implement rehabilitation with the Game System for Rehabilitation has a colorful screen and vivid audios. With the help of different equipment such as keyboard, mouse, gamepad, dance pad, wireless microphone and Kinect, the rehabilitation training could be done in extensive ways by individuals with autism and cerebral palsy. The system includes several items, therefore, individuals (both disabilities themselves and their trainers) can choose various rehabilitation games in diverse difficulty to fit them in different sites, mitigate the symptoms and improve the living qualities of people with disabilities. Different modalities and contents of the items in each program are given in Table 2:

Table 2. Items of game system for rehabilitation.

\begin{tabular}{|c|c|c|}
\hline NO. & Categories & Game Items \\
\hline \multirow{5}{*}{1} & \multirow{5}{*}{$\begin{array}{l}\text { Intellectual } \\
\text { Rehabilitation }\end{array}$} & Can you Tell the Difference \\
\hline & & Grasping the Order \\
\hline & & Eraser \\
\hline & & Graphics Memory \\
\hline & & Grasping the Order (Battle Mode) \\
\hline \multirow{6}{*}{2} & \multirow{6}{*}{$\begin{array}{l}\text { Upper Limbs } \\
\text { Rehabilitation }\end{array}$} & Throwing the Ball \\
\hline & & Picking Peaches \\
\hline & & Two Uses of One Mind \\
\hline & & Opening Sesame \\
\hline & & Gathering Treasure \\
\hline & & Stepping Up to Higher \\
\hline \multirow{4}{*}{3} & \multirow{4}{*}{$\begin{array}{l}\text { Lower Limbs } \\
\text { Rehabilitation }\end{array}$} & Beating the Drum \\
\hline & & Sumo \\
\hline & & Riding \& Shooting \\
\hline & & Treading Susliks \\
\hline \multirow{5}{*}{4} & \multirow{5}{*}{$\begin{array}{l}\text { Leisure } \\
\text { Activity }\end{array}$} & Goldfish Scooping \\
\hline & & Slotting Machine \\
\hline & & Bowling \\
\hline & & Bowling in Lines \\
\hline & & Red and White Bowling \\
\hline \multirow{6}{*}{5} & \multirow{6}{*}{$\begin{array}{l}\text { Basic } \\
\text { Perception \& } \\
\text { Cognition } \\
\text { Rehabilitation }\end{array}$} & Graphics Cognition \\
\hline & & Graphics Inference \\
\hline & & Words Cognitive Learning \\
\hline & & Numbers Cognitive Learning \\
\hline & & Smaller Muscle Training \\
\hline & & Perception Training \\
\hline
\end{tabular}

\subsection{Data collection}

The process of collecting data was conducted with 1 2 rehabilitation trainers selected from each community, school or hospital. Before the formal data collection, rehabilitation trainers selected were trained by the member of project team about the application of the Game System. Only when it is insured that trainers are acquainted with the system can they get down to intervening and train participants those who are responsible.

The first step of training is to assess each participant's ability, then, to make a personal specific plan of training. Training frequency and content were recorded by trainers during the training with the Game System. The training generally lasts 8 weeks, once or twice a week and 30 to 40 minutes at a time. After an eight-week training, an assessment with PEDI was conducted again. Meanwhile, collect those individual perspectives from parents, teachers, trainers as well as participants.

\subsection{Statistical analysis}

Many statistical analyses were performed with IBM SPSS software version 20.0. Three dimensions of PEDI (self-care, mobility and social function), disorder type (autism and cerebral palsy) and pre- and post-training tests were analyzed as independent variables. Results gained from PEDI were included in the analysis as dependent variables. Repeated-measures MANOVA and T test are used to analyze the effects of Game System of Rehabilitation.

\section{RESULTS}

\subsection{Overall effects}

Each participant was assessed using PEDI before and after the training with Game System for rehabilitation. In the analysis of the results obtained, we started from establishing the main existing effects and the interaction effects between two factors or among three factors. In this respect, we performed the analysis of repeated-measures MANOVA among the time (preand post- training), PEDI (self-care, mobility and social function) and disorder type (autism and cerebral palsy). The results are presented in Table 3:

In the part of functional skill, there significantly existed the main effects of factor time and PEDI, the interaction effects between each two factors, but not the interaction effects among three factors. And in the part of caregiver assistance, there also had the main significant effects of factor time and PEDI, the interaction effects between factor PEDI and disorder type, factor time and PEDI as well as the interaction effects among three factors, but not the interaction effects between factor time and disorder type.

In order to assess the improvement of participants with different disorders, ANOVA was used. The results of post hoc analysis and difference values between pre- and post- training gained in PEDI are shown in Table 4. It revealed that compared with autistic participants, people with $\mathrm{CP}$ made greater 
MATEC Web of Conferences

\begin{tabular}{|c|c|c|c|c|}
\hline & Factor & $M S$ & $F$ & $p$ \\
\hline \multirow{7}{*}{ Functional Skill } & Time & 6037.56 & 77.34 & $<0.01$ \\
\hline & PEDI & 3236.58 & 19.44 & $<0.01$ \\
\hline & Disorder Type & 97.28 & 0.19 & 0.667 \\
\hline & PEDI $\times$ Disorder Type & 6052.93 & 48.71 & $<0.01$ \\
\hline & Time $\times$ PEDI & 193.95 & 22.22 & $<0.01$ \\
\hline & Time $\times$ Disorder Type & 910.02 & 7.50 & $=0.01$ \\
\hline & Time $\times$ PEDI $\times$ Disorder Type & 9.53 & 0.76 & 0.473 \\
\hline \multirow{7}{*}{ Caregiver Assistance } & Time & 794.90 & 106.69 & $<0.01$ \\
\hline & PEDI & 4643.54 & 73.02 & $<0.01$ \\
\hline & Disorder Type & 36.75 & 0.25 & 0.623 \\
\hline & PEDI $\times$ Disorder Type & 712.56 & 22.47 & $<0.01$ \\
\hline & Time $\times$ PEDI & 7.79 & 4.82 & $<0.05$ \\
\hline & Time $\times$ Disorder Type & 30.08 & 2.63 & 0.114 \\
\hline & Time $\times$ PEDI $\times$ Disorder Type & 5.67 & 3.71 & $<0.05$ \\
\hline
\end{tabular}

Table 4. Comparison between autism and cerebral palsy.

\begin{tabular}{lllll}
\hline & PEDI & Autism & Cerebral palsy & $p$ \\
\hline \multirow{3}{*}{ Functional Skill } & Self-care & 5.45 & 9.78 & $=0.01$ \\
& Mobility & 1.65 & 8.64 & $<0.01$ \\
& Social Function & 7.33 & 12.72 & $<0.05$ \\
& Self-care & 2.65 & 3.58 & 0.166 \\
Caregiver Assistance & Mobility & 1.61 & 3.22 & $<0.05$ \\
& Social Function & 2.61 & 2.92 & 0.611 \\
\hline
\end{tabular}

Table 5. Differences between the pre- and post- training with autism

\begin{tabular}{|c|c|c|c|c|c|}
\hline \multirow{2}{*}{ PEDI } & & Pre-training & Post-training & \multirow{2}{*}{$t$} & \multirow{2}{*}{$p$} \\
\hline & & Mean \pm SD & Mean \pm SD & & \\
\hline \multirow{3}{*}{ Functional Skill } & Self-care & $43.14 \pm 10.17$ & $48.59 \pm 9.36$ & 8.41 & $<0.01$ \\
\hline & Mobility & $51.31 \pm 3.10$ & $52.96 \pm 1.99$ & 4.90 & $<0.01$ \\
\hline & Social Function & $27.80 \pm 11.50$ & $35.14 \pm 12.25$ & 9.30 & $<0.01$ \\
\hline \multirow{3}{*}{ Caregiver Assistance } & Self-care & $16.49 \pm 5.83$ & $19.14 \pm 4.59$ & 6.40 & $<0.01$ \\
\hline & Mobility & $26.14 \pm 5.68$ & $27.75 \pm 4.94$ & 4.12 & $<0.01$ \\
\hline & Social Function & $10.71 \pm 5.67$ & $13.31 \pm 4.76$ & 6.74 & $<0.01$ \\
\hline
\end{tabular}

T. spreadsheet at the level of $0.01=3.496$.

Table 6 . Differences between the pre- and post- training with cerebral palsy

\begin{tabular}{llllll}
\hline \multirow{2}{*}{ PEDI } & & \multicolumn{2}{c}{ Mean \pm SD } & & $p$ \\
\cline { 2 - 4 } & & Pre-training & Post-training & & $<$ \\
\hline \multirow{3}{*}{ Functional Skill } & Self-care & $35.42 \pm 14.83$ & $45.19 \pm 10.85$ & 5.644 & $<0.01$ \\
& Mobility & $36.47 \pm 17.76$ & $45.11 \pm 12.56$ & 4.916 & $<0.01$ \\
& Social Function & $37.47 \pm 19.45$ & $50.19 \pm 14.57$ & 5.199 & $<0.01$ \\
\multirow{2}{*}{ Caregiver Assistance } & Self-care & $13.81 \pm 6.50$ & $17.39 \pm 5.91$ & 6.606 & $<0.01$ \\
& Mobility & $20.50 \pm 9.29$ & $23.72 \pm 7.89$ & 4.724 & $<0.01$ \\
\hline
\end{tabular}

T. spreadsheet at the level of $0.01=3.582$.

progress in all aspects of functional skill and mobility of caregiver assistance. No significant differences were found in self-care and social function of caregiver assistance.

\subsection{Autism}

T test was used to contrast the autistic children' capacity with an eight-week training. From Table 5, it can be known that there had a significant enhancement on the ability of self-care, mobility and social functional, no matter with or without assistance from caregivers.

\subsection{Cerebral palsy}

In regard to the rehabilitation efficacy of children with $\mathrm{CP}, \mathrm{T}$ test is an optimal statistical approach to analyze whether the rehabilitation with Game System made 
sense. It is clear from Table 6 that the existence of statistically significant differences at the 0.05 significance level is between pre-training and post-training on all three dimensions of PEDI.

\section{DISCUSSION}

\subsection{Effectiveness}

The results of this study show that the children with the rehabilitation system based on Kinect had significant improvements on the skill of self-care, mobility and social Function, and the cerebral palsy performed better than the autism. One of the first symptoms that are commonly found in autistic children is the lack of imitation in social interaction (Dautenhahn \& Werry, 2014). Children with autism grow with limited acceptance or without any acceptance of something surrounding at all (Miskam et al. 2014). Severe autism has a low-functioning autism and it is most likely to be entirely unable to use spoken language. They experience the social interaction to be engaged in unpredictable behavior, aggression, frightening and property destruction (Koegel et al. 2012). So, during the training, the autistic was always asked to tell trainer names of these things after following the computer to learn which improves their expressed diction. At the same time, trainers tried to check their pronunciation, and this method greatly enhanced their communication ability. Teenagers love to play games, which can greatly improve their positivity, so they are more likely to be willing to communicate with others. In other research, the abilities in voice and vision detection recognition are crucial in human-robot interaction. Both elements will make the interaction more attractive towards children with autism. It also helps children with autism in improving their social and communication skills where the children need to respond. Imitation game of emotion also helps children in expressing feeling towards something according to the situation (Miskam et al. 2014). All of them bring their stronger communication ability when they finally return to the real society.

Compared with the autism, the cerebral palsy children have improved much more in self-care, mobility and social function with the rehabilitation system based on Kinect. The body program of rehabilitation is a benefit for their control ability, muscle power, tough and meticulous activity and body balanced. They would even break their limit to complete the game, which makes them feel well while obtain the best training effect. The rehabilitation system has a direct influence on their limbs and movements, it is an effect reflected on the self-care and mobility abilities. In addition, the training requests good understandings, which help them learn some social rules by learning the game rules. Meanwhile, it's also a great method to practice communicating. All of them help the children with cerebral palsy move forward in communicating.

\subsection{Prospects}

This system of recovery training game based on $\mathrm{Ki}$ nect Somatosensory interaction games has a broad prospect of application.

First, this system has broad applicable targets. Not only for the patients with autism and cerebral palsy, but also for those who with stroke, Alzheimer's disease and other special populations. The recovery training game refers to action, feeling, perception, attention, cognition and so on, so if someone has one of these demands, it's suitable.

Second, this system can be adopted in different institutions such as special education schools, rehabilitation institutions, welfare homes, hospitals, communities and families. Its colorful pictures, cute characters of cartoon and vivid animation greatly improve the children's enthusiasm of recovery training. Besides, it can apply for other handicapped people in hospitals as a new means of rehabilitation training. In addition, this system is suitable for PC, so people can do the training whenever they want.

At last, the combination of the traditional method of rehabilitation training and the system of recovery training game based on Kinect Somatosensory interaction games brings out a brand new method. As for the cerebral palsy, they can do the traditional rehabilitation training such as going down on their knees highly and alternately, walking while using the system of recovery training game based on Kinect, which obtains the best training effects. As for the autism, teachers can combine the traditional group class with the game system; teamwork is able to improve their ability of speech communication, imitation and social understanding.

No matter how the object, the place or the effect is, the system of recovery training game based on Kinect Somatosensory interaction games is a brand new method for handicapped people in our country.

\subsection{Insufficiencies and improvements}

This research based on the demand analysis of special population in our country is developing the rehabilitation system based on Kinect and choosing 87 persons to access roundly. However, there are still several insufficiencies:

1. The Rehabilitation system is a complex system with a variety of games and operations, but the sensitivity and accuracy as well as the frames of the game need to be enhanced because of the limit of time and technic.

2. Though we choose 87 children, the lack of comparisons such as the normal children narrows the extent of result.

In the further research, we can solve these problems from two parts. On one hand, we will consummate the game system to increase sensitivity and accuracy; on the other hand, we will strictly choose people, make the research normatively to increase the veracity of the 
Game System and provide a scientific and exact guidance of theory for the rehabilitation training of this special group.

\section{ACKNOWLEDGEMENT}

This study is one of achievements of the project: An interdisciplinary research of early detection, intervention, education for children with autism (12\&ZD229), which is sponsored by the key project of China Social Sciences Fund.

\section{REFERENCES}

[1] Bilde, P.E., Kliim-Due, M. \& Rasmussen, B. et al. 2011 Individualized, home-based interactive training of cerebral palsy chidren delivered through the Internet. $B M C$ Neurology, 11(1): 32.

[2] Bonnechere, B., Jansen, B. \& Salvia, P. et al. 2014 Validity and reliability of the Kinect within functional assessment activities: comparison with standard stereo photogrammetry. Gait \& Posture, 39(1): 593-598.

[3] Boutsika, E. 2014. Kinect in Education: A Proposal for Children with Autism. Procedia Computer Science, 27: 123-129.

[4] Burdea, G. 2002. Virtual rehabilitation-benefits and challenges. 1st International Workshop on Virtual Reality Rehabilitation (Mental Health, Neurological, Physical, Vocational) VRMHR. sn: 1-11.

[5] Burdea, G.C., Cioi, D. \& Kale, A. et al. 2013. Robotics and gaming to improve ankle strength, motor control, and function in children with cerebral palsy-A case study series. Neural Systems and Rehabilitation Engineering, IEEE Transactions on, 21(2): 165-173.

[6] Burke, J.W., McNeill, M.D.J. \& Charles, D.K. et al. 2009. Optimising engagement for stroke rehabilitation using serious games. Image and Vision Computing Journal, 25: 1085-1099.

[7] Chang, Y.J., Han, W.Y. \& Tsai, Y.C. 2013. A Kinect-based upper limb rehabilitation system to assist people with cerebral palsy. Research in developmental disabilities, 34(11): 3654-3659.

[8] Coleman, M.B., Heller, K.W. \& Cihak, D.F. et al. 2005. Using computer-assisted instruction and the nonverbal reading approach to teach word identification. Focus on Autism and Other Developmental Disabilities, 20(2): 80-90.

[9] Dautenhahn, K. \& Werry, I. 2014. Towards interactive robots in autism therapy: Background, motivation and challenges. Pragmatics \& Cognition, pp:1-35.

[10]De Mello Monteiro C B, Massetti T. \& Da Silva T D, et al. 2014. Transfer of motor learning from virtual to natural environments in individuals with cerebral palsy. Research in Developmental Disabilities, 35(10): 2430-2437.

[11]Dores, A.R., Carvalho, I.P., Barbosa, F. et al. 2012. Computer-assisted rehabilitation program-virtual reality (CARP-VR): A program for cognitive rehabilitation of executive dysfunction. Virtual and Networked Organizations, Emergent Technologies and tools, pp: 90-100.

[12] Golan, O. \& Baron-Cohen, S. 2006. Systemizing empathy: Teaching adults with Asperger syndrome or high-functioning autism to recognize complex emotions using interactive multimedia. Developmental and Psychopathology, 18: 591-617.

[13]James, S., Ziviani, J. \& Boyd, R. 2014. A systematic review of activities of daily living measures for children and adolescents with cerebral palsy. Developmental Medicine \& Child Neurology, 56: 233-244.

[14]Kandalaft, M.R. 2013. Virtual reality social cognition training for young adults with high-functioning autism. Journal of Autism and Developmental Disorders, 43: 34-44.

[15]Kim, D.A. \& Lee, J.A., Hwang, P.W. et al. 2012. The effect of comprehensive hand repetitive intensive strength training (CHRIST) using motion analysis in children with cerebral palsy. Annals of Rehabilitation Medicine, 36(1): 39-46.

[16]Koegel, L. Freden, R.M. \& Lang, R. et al. 2012. Interventions for Children with Autism Spectrum Disorders in Inclusive School Settings. Cognition and Behavioral Practice, pp: 401-412.

[17] Kroustallakis, G. 2005. Children with Special Needs. 6th ed. Athens (in Greek).

[18]LaBelle, K. 2011. Evaluation of Kinect Joint Tracking for Clinical and In-home Stroke Rehabilitation Tools, University of Notre Dame.

[19] Marie, B. \& Heidi, S. 2011. An intensive virtual reality program improves functional balance and mobility of adolescents with cerebral palsy. Pediatric Physical Therapy, 23(3): 258-266.

[20]Miskam, M.A., Msanin, N.S. \& Jamhuri, M.H. et al. 2014. Encouraging children with autism to improve social and communication skills through the game-based approach. Elsevier B.V. pp: 94-98.

[21] Moore, D., McGrath, P. \& Thorpe, J. 2000. Computer-aided learning for people with autism-a framework for research and development. Innovations in Education and Teaching International, 37(3): 218-228.

[22]Park, K.M., Ku, J. \& Choi, S.H. et al. 2011. A virtual reality application in role-plays of social skills training for schizophrenia: a randomized, controlled trial. Psychiatry Research, 189(2): 166-172.

[23]Parry, I., Carbullido, C. \& Kawada, J. et al. 2014. Keeping up with video game technology: Objective analysis of Xbox Kinect ${ }^{\mathrm{TM}}$ and PlayStation $3 \mathrm{Move}^{\mathrm{TM}}$ for use in burn rehabilitation. Burns: Journal of the International Society for Burn Injuries, 40(5): 852-859.

[24] Shane, H.C. \& Albert, P.D. 2008. Electronic screen media for persons with autism spectrum disorders: Results of a survey. Journal of Autism and Developmental Disorders, 38: 1499-1508.

[25] Sehaba, K., Estraillier, P. \& Lambert, D. 2005. Interactive educational games for autistic children with agent-based system. Lecture Notes in Computer Science, 3711: 422-432.

[26] Silver, M. \& Oakes, P. 2010. Evaluation of a new computer intervention to teach people with autism or Asper- 
ger syndrome to recognize and predict emotions in others. Autism, 5: 299-316.

[27] Wainer, A.L. \& Ingersoll, B.R. 2011. The use of innovative computer technology for teaching social communication to individuals with autism spectrum disorders. Research in Autism Spectrum Disorders, 5(1): 96-107.

[28] Weiss, P.L., Rand, D. \& Katz, N. et al. 2004. Video capture virtual reality as a flexible and effective rehabilitation tool. Neuroeng. Rehabil, 1: 12.

[29] Winkels, D.G.M., Kottink, A.I.R. \& Temmink, R.A.J. et

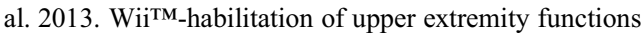
in children with Cerebral Palsy. An explorative study. Developmental Neurorehabilitation, 16: 44-51.

[30] Yeung, L.F., Cheng, K.C. \& Fong, C.H. et al. 2014 Evaluation of the Microsoft Kinect as a clinical assessment tool of body sway. Gait \& posture. 40(4): 532-538.

[31] Yeong, C.F., Melendez-Calderon, A. \& Gassert, R. et al. 2009. ReachMAN: A personal robot to train reaching and manipulation. Intelligent Robots and Systems. IROS 2009. IEEE/RSJ International Conference on. pp: 4080-4085. 